\title{
NORMATIVA SOBRE ESTUDIOS DE GÉNERO Y UNIVERSIDAD
}

\author{
ASUNCIÓN VENTURA FRANCH ${ }^{1}$ \\ Universitat Jaume I
}

Fecha de recepción: 7-9-2008.

Fecha de aceptación: 30-9-2008.

\section{Introducción}

Los estudios de género aparecen a finales de los años sesenta y principios de los 70 en Estados Unidos y Europa ${ }^{2}$. Estos estudios están vinculados -ése fue su inicio y continúa en gran medida- a lo que se denomina también el feminismo académico. Son las feministas las que, desde las distintas ramas científicas, han desarrollado estos estudios e investigaciones, que entre otras cosas han servido para fundamentar todas las medidas de igualdad que se han adoptado en el ámbito público y privado en los últimos años.

Cuando la Unión Europea y el Estado Español inician un proceso de legislación en relación con la igualdad y la no discriminación por razón de sexo, se apoyan básicamente en estos estudios que, en su mayor parte, han sido desarrollados fundamentalmente por mujeres. Se puede afirmar que existe una

1. Profesora titular de Derecho Constitucional de la Universitat Jaume I, de Castellón

2. BOSCH, Esperanza; FerRer, Victoria; AlzAMORA, Aina: El Laberinto patriarcal, Barcelona, Antropos, 2006, pp 66-72. Las profesoras explican el origen de estos estudios y las diferentes denominaciones de los mismos que se han sucedido en el tiempo, además de aportar una abundante bibliografía sobre el tema. En realidad, la denominación de los estudios de género es bastante reciente y, en la actualidad, se da una tendencia en el feminismo académico de recuperar todas las denominaciones que estos estudios han tenido desde los estudios de las mujeres (Women's Studies), Estudios Feministas y Estudios de género, en el mismo sentido se señala en VENTURA FRANCH, Asunción: «La integración de los estudios de las mujeres, feministas y de género en la universidad española», en Igualdad ¿para qué? A propósito de la Ley Orgánica para Igualdad efectiva de mujeres $y$ hombres, 2007, pp 521- 537. 
cantidad de conocimientos acumulados ${ }^{3}$, creados por el feminismo académico, en cualquiera de los ámbitos, que en estos momentos está sustentando, desde el punto de vista de la filosofía, la economía, la sociología y el derecho, todos los cambios que se están produciendo en los ámbitos políticos, sociales y económicos en relación con la igualdad entre mujeres y hombres.

Sin embargo, todos estos estudios representan un importante cuerpo teórico acumulado durante años que todavía las Universidades no integran dentro de lo que conocemos como el saber oficial. No es casual que la mayoría de estos trabajos e investigaciones hayan tenido que publicarse y buscar financiación diferente a la utilizada por el conocimiento clásico ${ }^{4}$. Todas las investigaciones sobre los estudios feministas y de género publicados en nuestro país constatan que la mayoría de ellos se ha publicado en editoriales específicas, mayoritariamente financiadas por organismos de igualdad. El saber oficial a día de hoy todavía cuestiona, en algunos casos abiertamente y en otros de forma indirecta, la validez de estos estudios, que, por cierto, incorporan la historia de la mitad de la humanidad a la vez que incluyen la existencia de dos sujetos en todos los ámbitos de la ciencia, donde hasta ahora se operaba con un solo sujeto, los hombres, aportando una visión omnicomprensiva frente a la parcialidad de la ciencia actual. A pesar de ello, y del avance que puede significar esta aportación a la ciencia, todavía a las personas que investigan desde esta perspectiva no se les otorga el reconocimiento académico que sí disfrutan las personas que lo hacen en el saber oficial.

En los últimos años, estos estudios han pasado a un primer plano, no sólo porque han fundamentado los cambios sociales producidos, sino también porque han sido objeto de regulación jurídica, y así las leyes, tanto autonómicas como estatales, relacionadas con la igualdad de mujeres y hombres, han incluido alguna referencia a los mismos, incluso la legislación en materia de universidades vincula los estudios de género con la enseñanza reglada.

Sin embargo, esta legislación, que representa un intento de incluir los estudios de las mujeres, feministas y del género en las enseñanzas universitarias, no ha tenido un efecto inmediato en la elaboración de los nuevos planes de estudio. Las razones de su ausencia son muy variadas y tienen diferentes orígenes pero en cualquier caso no hay que perder de vista que las leyes deben

3. Ballarin Domingo, Pilar; Gallego Mendez, M. Teresa; Martínez Benlloch, Isabel: Los Estudios de las Mujeres en las Universidades españolas 1975-91. Libro Blanco, Madrid, Ministerio de Asuntos Sociales. Instituto de la Mujer, 1995. OrTIZ GOMEZ, Teresa: Universidad y Feminismo II. Situación de los estudios de las mujeres en los años 90, Granada, Editorial Universidad de Granada, 1999.

4. Obras citadas en la nota 2. 
cumplirse y que tanto los ciudadanos como los poderes públicos están sujetos a la Constitución y al resto del ordenamiento jurídico.

\section{Necesidad de los estudios de género}

Los estudios de género, como se ha señalado anteriormente, tienen una larga tradición en el ámbito académico aunque su reconocimiento todavía no se ha realizado en toda su extensión y, quizá por este motivo se produce una cierta confusión respecto a su contenido, en muchas ocasiones de forma interesada y en otras por desconocimiento. Por ello, resulta conveniente concretar los criterios que deben presidir este tipo de estudios, ya que se dan algunas confusiones al respecto; una de las confusiones que se produce de manera casi generalizada es asociar estudios de género con mujeres. Todos aquellos estudios e investigaciones que hacen referencia a las mujeres se dice que son estudios e investigaciones de género. Pero, realmente, los estudios de género son algo muy diferente a la idea muy extendida de que hablar de género es hablar de mujeres. La palabra género ${ }^{5}$ denota la manera en que las relaciones entre los sexos se producen y se institucionalizan. En este sentido, los estudios de género analizan la relación de los géneros masculino y femenino, y la posición que ocupan cada uno de ellos, para el caso del género femenino de subordinación, y de dominación en el caso del género masculino. Si sólo se estudia la posición que ocupan las mujeres en la sociedad, el análisis es parcial y acientífico y también hasta cierto punto incomprensible; cualquier tipo de dominación no se puede entender si no se estudian las relaciones que se producen entre la persona subordinada y la persona dominante. Por ello, es necesario analizar las relaciones que se dan entre la persona subordinada y la persona dominante, qué relaciones de poder han generado, qué relaciones económicas, qué relaciones sociales, familiares y personales. De ahí que los estudios de género integren en el análisis esa idea más omnicomprensiva de las relaciones que han generado las diferentes asignaciones de funciones sociales en base al sexo.

En este sentido, cualquier estudio e investigación debe realizarse desde la perspectiva de género que supone considerar sistemáticamente las diferencias entre las condiciones, situaciones y necesidades respectivas de las mujeres y los hombres, y desvelar las formas sexuadas del saber. A partir de este análisis

5. COBO, R.: Cuadernos de Trabajo Social, XVIII (2005).

Feminismo/s 12, diciembre 2008, pp. 155-184 
se origina un nuevo saber que aporta una visión más omnicomprensiva frente a la parcialidad de la ciencia actual ${ }^{6}$.

Existen varias razones que justifican la necesidad de la inclusión de los estudios de género en la enseñanza reglada. En primer lugar, porque tal y como se ha señalado anteriormente, el saber oficial es un saber parcial y, por tanto, acientífico ${ }^{7}$ y es necesario ampliar los horizontes del saber y el conocimiento para un mejor desarrollo social y personal. En segundo lugar, porque ahora es el momento adecuado, teniendo en cuenta que con la creación del espacio europeo de educación superior y los acuerdos de Bolonia (1998), uno de los principios sobre los que se sustenta este nuevo espacio de educación superior es desarrollar Europa, y no podría entenderse este desarrollo si se prescinde de los estudios de género, dado que desde los años setenta la Unión Europea ha sido pionera en fomentar estudios e investigaciones introduciendo la perspectiva de género y desarrollando políticas de igualdad de género. Y la tercera y última razón obedece a que la consolidación de estos estudios en las instituciones académicas en general no es un acto de reconocimiento académico sin más, es el aprovechamiento de una contribución científica importante en la construcción de un mundo más igual y solidario, y que, entre otras cosas, ha servido para fundamentar todos los cambios que se han producido en relación con la igualdad de mujeres y hombres.

En la legislación española, tanto desde el gobierno central como desde las Comunidades autónomas, básicamente desde el año 2003 las diferentes leyes vienen contemplando de una u otra manera la necesidad de introducir la igualdad y no discriminación en el ámbito de la universidad y, en este sentido se pueden citar: en el ámbito de las Cortes Generales, la Ley Orgánica 1/2004, de 28 de diciembre, de Medidas de Protección Integral contra la Violencia de Género (en adelante LOMPIVG), la Ley Orgánica 3/2007, de 22 de marzo, para la igualdad efectiva de mujeres y hombres (en adelante LOI), Ley Orgánica 4/2007, de 12 de abril, por la que se modifica la Ley Orgánica 6/2001, de 21 de diciembre, de Universidades (en adelante LOM-LOU) y Real Decreto 1393/2007, de 29 de octubre, por el que se establece la ordenación de las enseñanzas universitarias oficiales.

Todas estas normas coinciden en la necesidad de integrar el principio de igualdad en las políticas de educación y en fomentar la enseñanza sobre el alcance y significado de la igualdad de mujeres y hombres en la educación

6. Puleo García, A.: «Moral de la transgresión, vigencia de un antiguo orden», Isegoría: Revista filosofía y política, 2003, pp. 245-252.

7. Primer Manifiesto de la Red Feminista de derecho constitucional, realizado en Biar los días 8 y 9 de junio (http://feministasconstitucional.org/node/25). 
superior, para que la formación en cualquier actividad profesional contribuya al conocimiento y desarrollo de los principios de igualdad entre mujeres y hombres.

De acuerdo con esta normativa, los principios generales para el diseño de los planes de estudio deben tener en cuenta que cualquier actividad profesional debe realizarse con el respeto a la igualdad de mujeres y hombres.

En la elaboración de los nuevos planes de estudio, según las directrices del Real Decreto 1393/2007, se introduce una serie de novedades y, entre ellas, el cumplimiento del principio de igualdad de oportunidades entre hombres y mujeres, que en el marco de la comunidad universitaria se incorpora explícitamente en la LOM-LOU (BOE 89, de 13 de abril de 2007). A tal efecto, las directrices de la $\mathrm{ANECA}^{8}$ para la elaboración de los nuevos planes de estudio de Grado señalan que los objetivos generales en el Grado deberán definirse teniendo en cuenta los derechos fundamentales y de igualdad de oportunidades entre mujeres y hombres, a la vez que en los planes de estudios en que proceda se deberá incluir enseñanzas relacionadas con los derechos fundamentales y de igualdad de oportunidades entre hombres y mujeres.

En base a todas estas consideraciones, los nuevos planes de estudio no pueden eludir la inclusión de enseñanzas que permitan conocer las causas de desigualdad, buscar soluciones para lograr la igualdad de mujeres y hombres y, por tanto, una sociedad en el siglo XXI más integrada y democrática.

Esta última cuestión es la que vamos a desarrollar en este artículo por cuanto entendemos que, en estos momentos, existe una legislación suficiente para poder implantar los estudios de género, en los nuevos planes de estudio que las universidades están modificando para su adaptación al nuevo espacio europeo de educación superior.

8. Artículo veintinueve de la LOM-LOU, establece: «El artículo 32 queda redactado del siguiente modo: «Artículo 32. Agencia Nacional de Evaluación de la Calidad y Acreditación. 1. Se autoriza la creación de la Agencia Nacional de Evaluación de la Calidad y Acreditación, de acuerdo con las previsiones de la Ley de Agencias Estatales para la mejora de los servicios públicos, a la que corresponden las funciones que le atribuye la presente Ley y la de elevar informes al ministerio competente en materia de universidades y al Consejo de Universidades sobre el desarrollo de los procesos de evaluación, certificación y acreditación en España, a cuyos efectos podrá solicitar y prestar colaboración a los órganos de evaluación que, en su caso, existan en las Comunidades Autónomas. 2. La Agencia Nacional de Evaluación de la Calidad y Acreditación desarrollará su actividad de acuerdo con los principios de competencia técnica y científica, legalidad y seguridad jurídica, independencia y transparencia, atendiendo a los criterios de actuación usuales de estas instituciones en el ámbito internacional». 


\section{Los estudios de género y la dispersión normativa}

A partir de las elecciones generales de 2004 se produce un cambio significativo en las políticas de igualdad. La igualdad de mujeres y hombres se incorpora a la agenda política y será uno de los principios que van a regir la actuación del nuevo gobierno 9 .

La primera ley que propone el gobierno es la LOMPIVG que aborda uno de los aspectos más problemáticos que tenía, y sigue teniendo, la sociedad para intentar dar protección a las mujeres víctimas de esta violencia. A ésta, siguieron otras normas ${ }^{10}$ que de alguna manera también estaban presididas por la idea de Igualdad, para regular diferentes ámbitos de la sociedad en los que la desigualdad de las mujeres resultaba contraria a los principios constitucionales.

Estas normas, en la medida en que pretenden abordar la situación de desigualdad de una manera integral, introducen como uno de los objetivos básicos para la igualdad la educación y la formación y, por ello, han incidido necesariamente en la universidad española como la institución de educación superior. Así, la universidad se ha visto afectada en estos últimos años por una serie de normas que han introducido una nueva perspectiva tanto en sus funciones como en la organización interna de la misma.

En términos generales se puede afirmar que la legislación de las universidades en relación a la igualdad como valor, principio y derecho reconocidos en la $C E^{11}$, ha tenido una trayectoria parecida al resto de las materias sobre las que se ha aplicado el principio de igualdad, esto es aplicar en un primer momento el concepto de igualdad formal. La Ley de reforma Universitaria ${ }^{12}$ se limitó a establecer los principios básicos de la igualdad formal, es decir, eliminar las discriminaciones desde la norma, pero en ningún momento se había planteado la necesidad de introducir el principio de igualdad material.

A este criterio general, se añade que la universidad parece un ámbito favorable a la igualdad, dado que tanto el acceso como la promoción se rigen por

9. El gobierno socialista a partir de las elecciones de 2004 ha reiterado la idea de que la igualdad de mujeres y hombres va a presidir la actuación de su política. Dicho extremo, se puede comprobar a través de la prensa escrita, virtual y en las comparecencias del gobierno y concretamente del presidente en el Congreso de los diputados.

10. Ley Orgánica 3/2007, de 22 de marzo, de igualdad efectiva entre mujeres y hombres. Ley Orgánica 4/2007, de 12 de abril, por la que se modifica la Ley Orgánica 6/2001, de 21 de diciembre, de Universidades. Ley Orgánica 1/2004, de 28 de diciembre, de Medidas de Protección Integral contra la Violencia de Género.

11. Ventura Franch, Asunción: Las mujeres y la Constitución Española de 1978, Madrid, Ministerio de Trabajo y Asuntos Sociales - Instituto de la Mujer, 1999.

12. Ley Orgánica 10/1983, de 25 de agosto, de la Reforma Universitaria (LRU). 
los principios de mérito y capacidad, por lo que en principio no se necesitaban medidas para fomentar la igualdad, aunque luego se ha demostrado que también en la universidad existen unos niveles de desigualdad considerables ${ }^{13}$.

Desde los años ochenta se han publicado numerosos estudios ${ }^{14}$ que han demostrado que no son realmente los méritos y la capacidad lo determinante para el acceso, la permanencia y la promoción dentro del ámbito universitario, y que en todas estas fases se produce un sesgo de género que perjudica claramente a las mujeres. Estos estudios introducen el debate en la universidad sobre tres cuestiones diferentes pero intrínsecamente relacionadas; la primera, sobre la necesidad de establecer políticas de igualdad en el ámbito universitario; la segunda, un cuestionamiento del saber oficial en la medida que lo consideran parcial; y la tercera, sobre la necesidad de introducir los estudios de las mujeres, feministas y del género ${ }^{15}$ en la enseñanza reglada. Esta última cuestión preocupa mucho, porque la falta de formación del alumnado en materia de igualdad y no discriminación, y el desconocimiento de la metodología para aplicar la perspectiva de género va a dificultar la puesta en marcha de las medidas que se adopten en este sentido ${ }^{16}$.

Estos debates van poco a poco tomando cuerpo, y si bien no tienen por sí mismos la fuerza suficiente para impulsar un cambio legislativo, resulta impensable que cualquier modificación legislativa que se produzca no los tenga en cuenta. La influencia de estos debates en los procesos legislativos se produce tanto a nivel del Estado, Cortes Generales, como a nivel de Comunidades

13. Numerosos estudios han demostrado este extremo, entre otros: libros blancos y ELIZONDO, Arantxa: «La presencia de mujeres y hombres en las universidades españolas», Aequalitas: Revista jurídica de igualdad de oportunidades entre mujeres y hombres, (21), 2007, pp. 25-34. Y la Unidad de Mujer y Ciencia del Ministerio de Educación y Universidades publicó un estudio: Académicas en Cifras, que muestra la posición de las mujeres y los hombres en la universidad (ver en: http://www.micinn.es/ciencia/umyc/ files/2007-academicas-en-cifras.pdf).

14. Los libros blancos, citados en la nota dos, iniciaron en España este tipo de análisis al que luego le han seguido muchos otros.

15. Esta nomenclatura de los Estudios es utilizada por parte del feminismo académico en la medida que representa una síntesis de lo que han sido estos estudios a través del tiempo. Ventura Franch, Asunción: «La integración de los estudios de las mujeres, feministas y de género en la universidad española», Igualdad ¿para qué? A propósito de la Ley Orgánica para Igualdad efectiva de mujeres y hombres, 2007, pp. 521- 537.

16. Se hace referencia a la formación de manera generalizada, porque formación en igualdad se está impartiendo en todas las universidades pero casi en exclusiva en el ámbito del voluntariado tanto por lo que respecta al profesorado como al alumnado; es decir, se imparte en doctorado, asignaturas de libre elección, posgrados, cursos, etc., propuestos por profesorado sensible a estos temas y a los que asiste alumnado también sensibilizado, pero el porcentaje representa una minoría (ver: Libros blancos).

Feminismo/s 12, diciembre 2008, pp. 155-184 
autónomas, dado que las competencias en educación están transferidas en muchas de las CCAA. Cabe destacar que en el ámbito de las CCAA desde el año 2003 se vienen aprobando leyes de igualdad ${ }^{17}$ en las que se incorporan aspectos relacionados con la enseñanza y las universidades en la medida que las CCAA van adquiriendo competencias en materia de universidades.

La primera ley en el ámbito estatal que contempla medidas de igualdad en la Universidad es la LOMPIVG; pretende ser una ley integral y por ello abarcar los diferentes ámbitos en que se puede incidir para eliminar la violencia y proteger a las víctimas. Uno de los aspectos que la ley aborda es el sistema educativo $^{18}$, así en el título primero incluye un artículo (artículo 4) dedicado a los principios y valores que deben presidir el sistema educativo, y aunque en un primer momento en el proyecto de ley presentado en el Congreso se deja fuera el ámbito universitario ${ }^{19}$, posteriormente se incluye un nuevo apartado, séptimo, en el que se contempla que las universidades incluirán y fomentarán en todos los ámbitos académicos la formación, docencia e investigación en igualdad de género y no discriminación de forma trasversal.

Esta ley, que como ya es conocido se aprobó sin votos en contra, no tuvo en este aspecto ninguna consecuencia inmediata en el ámbito de las universidades. Todo siguió igual y no se adoptó ninguna medida para aplicarla. No obstante, la aprobación de la ley motivó un debate en el seno de las universidades $^{20}$, auspiciado por el feminismo académico y las diferentes redes de

17. Ley Foral 33/2002, de 28 de noviembre, de fomento de la igualdad de oportunidades entre mujeres y hombres (Navarra); Ley 1/2003, de 3 de marzo, de igualdad de oportunidades entre mujeres y hombres en Castilla y León (Castilla y León); Ley 9/2003, de 2 de abril, para la igualdad entre mujeres y hombres (Comunidad Valenciana); Ley 7/2004, de 16 de julio, gallega para la igualdad de mujeres y hombres (Galicia); Ley del Parlamento Vasco 4/2005, de 18 de febrero, para la igualdad de mujeres y hombres (País Vasco); y Ley 7/2007, de 4 de abril, para la igualdad entre mujeres y hombres, y de protección contra la violencia de género en la Región de Murcia (Murcia).

18. La educación y la formación son indispensables para modificar actitudes.

19. La elaboración de la LOMPIVG introdujo una novedad en nuestro país en el proceso legislativo, la presencia de personas expertas en el Congreso de los diputados en la comisión correspondiente. Así, el presidente del Congreso nombró a una serie de expertas para comparecer en la Comisión de Trabajo y Asuntos Sociales con el fin de recabar su opinión con respecto al proyecto de ley. Algunas de estas expertas plantearon la necesidad de incorporar en las enseñanzas universitarias la igualdad y no discriminación. En este sentido ver la intervención en la Comisión de Trabajo y Asuntos Sociales el 09/09/2004 cuando comparece la profesora Asunción Ventura Franch (219/000043). Posteriormente, Esquerra Republicana propone una enmienda en la que se introduce el apartado 7 del artículo 4 y, finalmente se aprueba por unanimidad.

20. Se adjuntan como Anexo las conclusiones de algunos de estos debates. 
investigación feministas y de género, acerca de la necesidad de aplicar la ley en las universidades.

En el año 2007, se aprueba la LOI; esta ley tampoco puede obviar el debate acerca de la importancia de la educación y la formación, y por ello incorpora en el Título II, dedicado a las Políticas Públicas de Igualdad, el art. 25 que regula la igualdad en el ámbito de la educación superior ${ }^{21}$.

A partir, de la aprobación de esta ley existe un ámbito -el universitarioregulado por dos leyes orgánicas en las que se incorpora el debate planteado por el feminismo académico sobre las tres temáticas, anteriormente señaladas, políticas públicas, investigación y docencia.

De la primera ley (LOMPIVG) a la segunda (LOI) transcurren dos años y algunos meses y, se puede afirmar que en este tiempo, por parte de la administración universitaria se han adoptado pocas medidas ${ }^{22}$ para el cumplimiento de lo establecido en el art. 4.7 de la LOMPIVG.

Si se realiza un análisis comparado de los artículos ${ }^{23}$ que hacen referencia a la universidad en cada una de las leyes referenciadas -el art. 4.7 de LOMPIVG

21. Artículo 25. La igualdad en el ámbito de la educación superior: 1. En el ámbito de la educación superior, las Administraciones públicas en el ejercicio de sus respectivas competencias fomentarán la enseñanza y la investigación sobre el significado y alcance de la igualdad entre mujeres y hombres. 2. En particular, y con tal finalidad, las Administraciones públicas promoverán: a) La inclusión, en los planes de estudio en que proceda, de enseñanzas en materia de igualdad entre mujeres y hombres. b) La creación de postgrados específicos. c) La realización de estudios e investigaciones especializadas en la materia.

22. El informe Ejecutivo sobre la Evaluación de la aplicación de la Ley Orgánica (www. ministeriodeigualdad.es) apartado 1.2.

23. Artículo 4 de la LOMPIVG, establece: «Principios y valores del sistema educativo.1. El sistema educativo español incluirá entre sus fines la formación en el respeto de los derechos y libertades fundamentales y de la igualdad entre hombres y mujeres, así como en el ejercicio de la tolerancia y de la libertad dentro de los principios democráticos de convivencia. Igualmente, el sistema educativo español incluirá, dentro de sus principios de calidad, la eliminación de los obstáculos que dificultan la plena igualdad entre hombres y mujeres y la formación para la prevención de conflictos y para la resolución pacífica de los mismos.2. La Educación Infantil contribuirá a desarrollar en la infancia el aprendizaje en la resolución pacífica de conflictos.3. La Educación Primaria contribuirá a desarrollar en el alumnado su capacidad para adquirir habilidades en la resolución pacífica de conflictos y para comprender y respetar la igualdad entre sexos.4. La Educación Secundaria Obligatoria contribuirá a desarrollar en el alumnado la capacidad para relacionarse con los demás de forma pacífica y para conocer, valorar y respetar la igualdad de oportunidades de hombres y mujeres.5. El Bachillerato y la Formación Profesional contribuirán a desarrollar en el alumnado la capacidad para consolidar su madurez personal, social y moral, que les permita actuar de forma responsable y autónoma y para analizar y valorar críticamente las desigualdades de sexo y fomentar la igualdad real y efectiva entre hombres y mujeres.6. La Enseñanza para las personas adultas incluirá entre sus objetivos desarrollar actividades en la resolución

Feminismo/s 12, diciembre 2008, pp. 155-184 
y el art. 25 de la LOI-, se puede afirmar que la norma que contiene una mayor exigencia y obligación es la ley contra la violencia de género, sin embargo la LOI mantiene un tono más incentivador, más de fomentar y por tanto, menos imperativo. Parece que la ley de igualdad (LOI) se inclina por desarrollar uno de los aspectos que ya regula la ley contra la violencia de género (LOMPIVG), el que se refiere a las acciones de promoción, pero no tiene en cuenta la parte más imperativa.

Para facilitar la comparación y poder determinar el alcance de los preceptos, a continuación se copian los textos de los artículos 4.7 y 25.1 de la ley contra la violencia de género y la ley de igualdad y se sombrean las palabras en las que se centra la cuestión de establecer políticas regulativas o políticas de promoción.

Así:

Las universidades incluirán y fomentarán en todos los ámbitos académicos la formación, docencia e investigación en igualdad de género y no discriminación de forma transversal (Art. 4.7 de la LOMPIVG).

En el ámbito de la educación superior, las administraciones públicas en el ejercicio de sus respectivas competencias fomentarán la enseñanza y la investigación sobre el significado y alcance de la igualdad entre mujeres y hombres. (art.25.1 de la LOI)

El primer texto, el art .4.7 de la LOMPIVG, introduce dos tipos de obligaciones, la de incluir en todo lo relativo a la docencia, formación e investigación la igualdad de género y no discriminación de forma transversal y, además, añade la obligación de fomentar.

Si tenemos en cuenta el significado de la palabra incluir ${ }^{24}$, resulta bastante evidente que la ley establece un mandato a las universidades para que en el desarrollo de estas materias y, sobre todo, en el ámbito de sus competencias, incluyan estos postulados. También establece la obligación de fomentar ${ }^{25}$ y, en ese sentido, se puede entender como promover acciones para incrementar los estudios y las investigaciones en esta materia. Por tanto, cabe resaltar estos dos mandatos: uno más concreto, el de incluir en la formación, la docencia y la investigación el principio de igualdad y no discriminación por razón de

pacífica de conflictos y fomentar el respeto a la dignidad de las personas y a la igualdad entre hombres y mujeres.7. Las Universidades incluirán y fomentarán en todos los ámbitos académicos la formación, docencia e investigación en igualdad de género y no discriminación de forma transversal».

24. La REA en la versión última define el significado de incluir como contener a otra o llevarlo implícito.

25. La REA en la versión última define el significado de fomentar como promover, impulsar o proteger algo. 
sexo; y otro, la obligación de fomentar que resulta mucho más inconcreto, en la medida que la ley no señala ninguna acción de promoción, y por lo tanto tendrían que ser la propias universidades las que concretaran dichas acciones.

En lo que respecta a la docencia y a la formación, esta obligatoriedad conlleva la necesidad de que en los planes de estudio y en la programación de la formación se garanticen la enseñanza sobre el significado y alcance de la igualdad de género. El mismo tratamiento se debía aplicar a la investigación tanto en los planes nacionales como autonómicos y en el de las propias universidades ${ }^{26}$.

En lo que respecta a la LOI, si se observa detenidamente el texto del art. 25.1 , se puede señalar que esta ley sólo contempla una de las obligaciones ya señaladas en la LOMPIVG y dirigida a las administraciones públicas, por tanto no sólo atañe a las universidades sino también a todas la administraciones públicas; se sobreentiende que se trata de todas aquellas que tengan competencia en la materia. Dicha obligatoriedad está referida a las acciones de promoción, pero además concreta algunas de estas acciones (art. 25.2). Así, se concreta en: «... realizar actividades de promoción en la enseñanza y en la investigación, sobre el alcance y el significado de la igualdad entre mujeres y hombres». En el apartado segundo ${ }^{27}$ establece tres medidas que deben entenderse como mínimas porque de acuerdo con esta redacción no se impide que las administraciones públicas puedan adoptar otras medidas de fomento o promoción además de las allí señaladas. La LOI concreta algunas acciones que deben realizar las administraciones para fomentar la enseñanza y la investigación sobre el significado y alcance de la igualdad entre mujeres y hombres.

Ambas leyes coinciden en la necesidad de fomentar y promocionar acciones para la igualdad y, en este sentido se puede afirmar que la LOMPIVG establece un mandato de carácter general y la LOI una concreción mínima de las acciones a adoptar por las respectivas administraciones, no obstante no se excluye la posibilidad de adoptar otro tipo de medidas de fomento o promoción en la enseñanza y la investigación.

Se puede constatar que, aunque aparentemente no se haya establecido una conexión directa entre ambas leyes, lo cierto es que existe una clara relación

26. Muchos de los proyectos de investigación son convocatorias estatales, autonómicas y europeas.

27. Artículo 25. La igualdad en el ámbito de la educación superior: 2. En particular, y con tal finalidad, las Administraciones públicas promoverán: a) La inclusión, en los planes de estudio en que proceda, de enseñanzas en materia de igualdad entre mujeres y hombres. b) La creación de postgrados específicos. c) La realización de estudios e investigaciones especializadas en la materia. 
entre ellas, sobre todo en lo que respecta a la acción de promover. Pero la cuestión radica en que la LOI no ha incorporado el sentido obligatorio que comporta la palabra incluirán, entendida, como se ha señalado anteriormente, como un mandato a las universidades para concretar, al igual que ha hecho con la acción de promover, cómo o qué medidas se deben adoptar para llevar a cabo esa obligatoriedad que se desprende de la palabra incluirán en la formación, docencia e investigación en igualdad de género y no discriminación de forma transversal.

Esta falta de conexión podría, en una primera lectura, interpretarse como una contradicción entre ambas leyes, por cuanto de la primera se deduce que toda la formación, toda la docencia y toda la investigación deben ser atravesadas $^{28}$ (transversal) por la igualdad y no discriminación. Sin embargo, en relación a la docencia la LOI se limita a regular que el significado y alcance de la igualdad entre mujeres y hombres se incluirán, siempre que las administraciones publicas así lo promuevan, en los planes de estudio en que proceda, sin determinar en cuáles sí y en cuáles no, ni qué criterios deben aplicarse para incluir o no estas enseñanzas en los planes de estudio.

La LOI al contrario de lo que contempla la LOMPIVG tampoco hace referencia a la formación, que no debe ser entendida como sinónimo de docencia. En el concepto de formación estarían incluidos los propios docentes en la medida que también pueden formarse como viene siendo habitual, sobre todo, en los últimos tiempos en que las universidades se preocupan de la formación del profesorado novel y también de fomentar el necesario reciclaje del profesorado.

La LOI también se refiere a los posgrados, que, como es bien conocido, son la última fase del proceso de la enseñanza reglada, producto básicamente de la adaptación de las enseñanzas al espacio europeo de educación superior. Los posgrados oficiales, o de acuerdo con la nueva normativa ${ }^{29}$ posgrados universitarios, son parte de la enseñanza reglada y por ello estarían incluidos en la docencia y, en ese caso, también de acuerdo con la LOMPIVG, todos y cada

28. Transversalidad: «La integración sistemática de las respectivas situaciones, prioridades y necesidades de mujeres y hombres en todas las políticas de cara a promover la igualdad entre mujeres y hombres y movilizar todas las políticas y medidas generales específicamente para el propósito de alcanzar la igualdad de forma activa y abierta, teniendo en cuenta, tanto a nivel de la planificación, como en el de la implimentación, monitorización y evaluación, los efectos sobre las respectivas situaciones de mujeres y hombres» (Comission Comunication, COM (96) 67 FINAL, 21.2.1996).

29. Real Decreto 56/2005, de 21 de enero, por el que se regulan los estudios universitarios oficiales de Posgrado y Real Decreto 1393/2007, de 29 de octubre, por el que se establece la ordenación de las enseñanzas universitarias oficiales. 
uno de ellos deberían incluir la igualdad y no discriminación en una perspectiva transversal. Sin embargo, la LOI se refiere a la creación de posgrados específicos, relacionados con la materia de igualdad de mujeres y hombres, cuestión que no resulta incompatible con la obligatoriedad de introducir en todo los posgrados la perspectiva de género, porque, efectivamente, puede necesitarse una especialización tanto en la vertiente profesional como investigadora en esta materia.

Pero ello no impide la obligatoriedad establecida en la LOMPIVG de que en todos los posgrados se analice el significado de la igualdad de mujeres y hombres, sobre todo porque es necesario que cualquier persona que estudie en la universidad tenga una mínima formación en este sentido, para capacitarla y poder así desarrollar las competencias y habilidades necesarias con el fin de intervenir en los procesos de desigualdad cualquiera que sea su profesión.

Del análisis realizado se puede afirmar que, por una parte, se da una clara sintonía entre ambas leyes, sobre todo en lo que respecta a la promoción y a fomentar la docencia y la investigación, pero también se puede constatar la falta de armonía entre ambas en cuanto a la obligatoriedad de incluir en la docencia, formación e investigación de forma transversal la igualdad y no discriminación.

De lo anteriormente señalado podemos deducir que se trata de leyes complementarias y coincidentes en cuanto a la necesidad de promoción de esta temática. Sin embargo, resulta claramente meridiano que hay una parte del mandato contemplado en el art.4.7 de LOMPIVG que no se menciona en la LOI.

\section{La Ley Orgánica de Universidades: igualdad de mujeres y hombres}

La Ley Orgánica 6/2001, de 21 de diciembre, de Universidades ha sido modificada por la Ley Orgánica 4/2007, de 12 de abril. El objetivo básico de la modificación ha sido los cambios sociales producidos en los cinco años de vigencia de la LOM-LOU, y sobre todo los acuerdos en política de educación superior en Europa y el impulso que desde la Unión Europea se pretende dar a la investigación. La ley, también, pretende introducir los avances que se han realizado en otros aspectos y hace una especial mención a la universidad como una institución transmisora de valores. Y, en concreto, alude a la igualdad de mujeres y hombres como uno de los valores que debe desarrollar la universidad para contribuir al reto de una sociedad actual. En este sentido, el Preámbulo señala: «... el papel de la universidad como transmisor esencial de valores y entre ellos señala alcanzar una sociedad tolerante e igualitaria, en la 
que se respeten los derechos y libertades fundamentales y de igualdad entre hombres y mujeres» (Preámbulo párrafo 12).

En relación con los valores la LOM-LOU los asume como objetivos propios de la universidad (Preámbulo párrafo 12) y concreta algunas políticas para hacerlos efectivos; así:

- El establecimiento de sistemas que permitan alcanzar la paridad en los órganos de representación.

- Una mayor participación de la mujer en los grupos de investigación.

- La creación de programas específicos sobre la igualdad de género.

- La creación de programas específicos de ayuda a las víctimas del terrorismo

- Impulsar políticas activas para garantizar la igualdad de oportunidades a las personas con discapacidad.

Algunos de estos valores anunciados en el preámbulo se concretan en el texto de la ley adoptando acciones para que puedan ser una realidad, y en este sentido el artículo diez de la nueva ley que modifica el artículo 13 de la anterior y, en relación a los órganos de gobierno y representación de las Universidades Públicas, añade un nuevo párrafo en el que regula que los estatutos establecerán las normas electorales aplicables, las cuales deberán propiciar en los órganos colegiados la presencia equilibrada entre mujeres y hombres.

También en el ámbito de la investigación, la nueva ley, en concordancia con los valores establecidos, modifica la ley anterior (el art. 39 modifica el art. 41.1) y, entre otros objetivos, señala que la universidad desarrollará una investigación para garantizar el fomento y la consecución de la igualdad, a la vez que incide en la necesidad de que las mujeres participen en los grupos de investigación. En cuanto a propiciar una mayor participación de las mujeres en los grupos de investigación, la nueva ley en el art. cuarenta y dos añade dos apartados al art. 41 de la ley anterior, y en el apartado 4), regula: «Se promoverá que los equipos de investigación desarrollen su carrera profesional fomentando una presencia equilibrada entre mujeres y hombres en todos sus ámbitos». También, en relación a los objetivos de la investigación ${ }^{30}$ que desarrolle la universidad, señala el deber de contribuir al desarrollo de la sociedad teniendo en cuenta el progreso económico y social, y un desarrollo responsable equitativo y sostenible, así como garantizar el fomento y la consecución de la igualdad.

Un aspecto que cabe destacar es la idea, como se ha dicho anteriormente, que recoge en el preámbulo, y que señala a la universidad como transmisora

30. Artículo 39 de la LOM-LOU que modifica el apartado 1del artículo 41 de la LOU. 
esencial de valores ${ }^{31} \mathrm{y}$, entre otros, contempla la igualdad de mujeres y hombres. Pero la cuestión radica en concretar a través de qué mecanismos la universidad puede transmitir los valores. En primer lugar, cabe afirmar que lo suyo sería que la transmisión de los valores debe producirse en el desarrollo de sus funciones, que, de acuerdo con lo que señala el art. 1.2 de la LOM-LOU, sería a través de la creación, desarrollo, transmisión y crítica de la ciencia, de la técnica y de la cultura, la preparación para el ejercicio de actividades profesionales, la transferencia y difusión del conocimiento.

Las cuatro funciones que señala la ley deben estar atravesadas por el valor de alcanzar una sociedad tolerante e igualitaria en la que se respeten los derechos y libertades fundamentales y de igualdad entre mujeres y hombres. Por tanto, las universidades deben establecer acciones para que estos valores impregnen todas y cada una de las funciones señaladas en la ley.

También La LOM-LOU recoge los principios que deben regir la organización interna de las universidades y, entre otros, señala la paridad en los órganos de representación y una mayor participación en los grupos de investigación. Los poderes públicos deben remover los obstáculos que impidan a las mujeres alcanzar una presencia en los órganos de gobierno de las universidades y en el nivel más elevado de la función pública docente e investigadora acorde con el porcentaje que representan entre los licenciados universitarios.

La LOM-LOU marca algunas acciones para la consecución de la igualdad de mujeres y hombres, como la creación de programas específicos sobre igualdad de género, de ayuda a las víctimas del terrorismo y el impulso de políticas de activas para garantizar la igualdad de oportunidades a las personas con discapacidad.

Sin embargo, la ley no concreta ninguna acción, más allá de programas específicos, en relación con la docencia, por cierto, una de las funciones más importantes de la universidad que conduce a la preparación para el ejercicio de actividades profesionales que exijan la aplicación de conocimientos y métodos científicos para la creación artística. Sin duda, esta función está directamente vinculada con la docencia reglada y por tanto con el diseño y los contenidos de los planes de estudio sobre los que se tendrán que aplicar o concretar los valores señalados en el preámbulo de la ley y, en concreto, la igualdad de mujeres y hombres.

31. Existe una abundante literatura en relación con la concreción de los valores, sin entrar en la polémica de la diferencia entre principios y valores en relación a la CE ya señaladas en otra obra VentuRA FRANCH, Asunción: Las Mujeres y la constitución Española..., op. cit., p.154 y ss.

Feminismo/s 12, diciembre 2008, pp. 155-184 
La docencia es una de las funciones básicas de la universidad en las que se va a preparar a los futuros profesionales para el desarrollo de actividades que exijan la aplicación de conocimientos y métodos científicos.

La LOM-LOU distingue dos tipos de enseñanzas ${ }^{32}$ : las que conducen a la obtención de los títulos oficiales y con validez en todo el territorio nacional y, las enseñanzas que conducirán a títulos no oficiales. En relación con las enseñanzas oficiales, el gobierno establecerá las directrices y las condiciones para la obtención de los títulos universitarios de carácter oficial ${ }^{33}$ que serán expedidos en nombre del Rey por el rector de la Universidad.

A pesar de todo lo señalado anteriormente, en el ámbito de la docencia, la LOM-LOU no introduce ninguna política relacionada con alcanzar una sociedad tolerante e igualitaria, en la que se respeten los derechos y libertades fundamentales y de igualdad entre hombres y mujeres (Preámbulo párrafo 12). Sólo cabe mencionar que en el artículo noventa de la nueva ley que modifica la Disposición adicional vigésima cuarta, en el apartado 5, regula que todos los planes de estudio propuestos por las universidades deben tener en cuenta que la formación en cualquier actividad profesional debe realizarse desde el respeto y la promoción de los derechos humanos y los principios de accesibilidad universal y diseño para todos. Y esta referencia, se contextualiza en la inclusión en las universidades de las personas con discapacidad.

Fuera de esta precisión, que luego no se concretará en las directrices establecidas por el Gobierno ${ }^{34}$, la LOM-LOU no contempla los criterios que deben presidir la elaboración de los planes de estudio, sino que deja en manos del gobierno el establecimiento de los mismos, sobre los que el gobierno en el desarrollo reglamentario (RD 1393/2007) ha concretado en dos fases, una a priori (RD 1393/2007) en el momento de la elaboración de los planes de estudio $\mathrm{y}$, otra posteriori, al efecto de comprobar si los planes que se presentan para su aprobación al organismo competente $\mathrm{ANECA}^{35}$, han incorporado los criterios fijados por el gobierno.

La concreción de los valores en relación con la función docente de las universidades queda atribuida a la competencia del gobierno para determinar las directrices y las condiciones para la elaboración de los planes de estudio, pero el gobierno no puede apartarse de los mandatos legales y debe concretar

32. Artículo 30 de la LOM modifica el art. 34 de la LOU.

33. Artículo 31 de la LOM que modifica el art. 35 de la LOU.

34. En el Real Decreto 1393/2007, de 29 de octubre, por el que se establece la ordenación de las enseñanzas universitarias oficiales

35. Agencia Nacional de Evaluación de la Calidad y Acreditación (art. 29 de la LOM-LOU).

Feminismo/s 12, diciembre 2008, pp. 155-184 
unas directrices que se adecuen a los valores, principios y reglas prescriptivas señaladas en las leyes.

En este sentido, y en relación al tema que nos ocupa, las directrices deben contemplar los valores que incorpora la LOM-LOU en el preámbulo, así como el mandato expreso formulado en la LOMPIVG y, concretar cómo se va a incorporar en la docencia los derechos fundamentales y la perspectiva de género, además de concretar los mecanismos para la verificación de su inclusión de tal manera que se pueda verificar la validez o no de un plan de estudios de acuerdo con las directrices.

\section{La perspectiva de género en las directrices para}

\section{la ordenación de las enseñanzas universitarias oficiales}

El gobierno, de acuerdo con la potestad que le otorga la LOM-LOU debe marcar las directrices para la elaboración de los planes de estudio, y en base a ello dictar un RD en el que establece las reglas fundamentales sobre las que van a girar las enseñanzas universitarias. Pero a la hora de establecer estas reglas, el gobierno debe tener en cuenta la regulación con rango de ley que existe sobre la materia en cuestión y, también la existencia de una pluralidad de normas que regulan la misma materia: la ley integral, la ley de igualdad y la ley orgánica de universidades, todas ellas leyes orgánicas. Estas normas, como se ha señalado anteriormente, establecen dos tipos de mandatos con respecto a la inclusión en las enseñanzas universitarias de una materia vinculada a la igualdad; un primer mandato a las universidades para incluir en todos los ámbitos académicos la formación, docencia e investigación en igualdad de género y no discriminación de forma transversal (art.4.7 de la LOMPIVG) y, otro, de fomento señalado también en el art.4.7 de la referida ley y en el art.25.1 de la LOI. Así, se contemplan dos tipos de acciones que el gobierno debía desarrollar en el RD 1393/2007 con el fin de garantizar que en las universidades se impartan estas materias y desarrollar en las directrices el cumplimiento de estos mandatos. A ello, se une que la ley de Universidades (LOM-LOU) contempla la igualdad de mujeres y hombres como un valor que debe concretarse a través de las funciones de la propia universidad.

En este sentido, el RD 1393/2007 debería haber incorporado, por una parte, el cumplimiento estricto de la ley, y además, haber convertido en operativos $^{36}$ los preceptos legales que por sí mismos resultaran de difícil aplicación. Este sería el caso por cuanto que las leyes establecen unos principios que necesitan una concreción para poder desarrollarse. Esta apreciación es

36. Balaguer Callejón F: Fuentes del Derecho, Tomo II, Madrid, Tecnos, 1992, p. 117. 
confirmada por el propio RD 1393/2007 que en la parte expositiva señala que «...el presente real decreto, siguiendo los principios sentados por la citada ley (en referencia a la LOM-LOU)».

El desarrollo del RD 1393/2007 se vincula a la LOM-LOU pero no ha tenido en cuenta el contenido de las dos leyes de referencia (LOMPIVG y la LOI). La cuestión radica en saber por qué el gobierno en el ejercicio de la potestad reglamentaria no desarrolla de forma conjunta todos aquellos mandatos que señalan las leyes, en materia de formación y docencia universitaria y que, necesariamente, requieren una concreción para poder llevarlos a la práctica. Quizá el gobierno pensara en desarrollar otros reglamentos ${ }^{37}$ vinculados a cada una de las leyes. En cualquier caso, el RD 1393/2007 no puede contradecir lo establecido en las leyes y, además, es necesaria una cierta homogeneización por cuanto las leyes, todas orgánicas, regulan una materia común. Quizá, lo ideal hubiera sido que la LOM-LOU unificara los criterios establecidos en las anteriores leyes.

El gobierno en el desarrollo del RD 1393/2007 ha adoptado una posición más mono-color, como se señala por algunos autores ${ }^{38}$, dejando atrás la posición sustentada por las minorías parlamentarias, no del todo asumidas por la mayoría parlamentaria y que por condicionamientos varios y, sobre todo, con el fin de obtener acuerdos parlamentarios, no tuvo más remedio que aceptar como ocurrió con la LOMPIVG ${ }^{39}$, dado que el texto del art. 4.7 de esta ley es la que regula de forma más imperativa la inclusión de los estudios de género en la enseñanza reglada.

El RD 1393/2007, no tiene en cuenta lo regulado en la LOMPIVG ni los valores señalados en la LOM-LOU e intenta establecer una serie de medidas para poder ordenar las enseñanzas universitarias, y así, concreta la estructura de las enseñanzas, estableciendo las enseñanzas de grado, posgrado y doctorado, a la vez que determina el proceso de verificación y acreditación de los títulos, establece el número de créditos, determina las materias para todos los estudios obligatorias (que constan en los anexos), materias básicas por rama de conocimiento, que necesariamente figuraran en al menos 36 créditos de formación básica, vinculadas a las materias de la rama a la que se adscriba el

37. Sin embargo, este supuesto a pesar de ser posible no se ha llevado a la práctica.

38. Balaguer Callejón, F: Op. cit., p. 119.

39. En concreto, el apartado 7 del artículo 4 de la Ley Orgánica 3/2007, de 22 de marzo, para la igualdad efectiva de mujeres y hombres, se corresponde con el texto presentado en la enmienda núm. 349 por el Grupo Parlamentario de Esquerra Republicana. Boletín Oficial de las Cortes Generales. Congreso de los Diputados. VIII Legislatura de 19 de octubre de 2004, p.155. 
Plan de Estudios. El resto hasta los sesenta créditos podrán ser de las materias que figuran en la rama correspondiente, o bien de las materias de las otras ramas, incorporando la idea de la multidisciplinariedad.

Sin embargo, resulta curioso que en ninguna de las ramas se haya incluido una materia que coincida con los derechos humanos, etc. A pesar, que en la exposición de motivos del RD 1393/2007 se señala que la formación en cualquier actividad profesional debe contribuir al conocimiento y desarrollo de los derechos humanos. La afirmación realizada en la exposición de motivos del RD 1393/2007 concuerda con los valores establecidos en la LOM-LOU y con la LOMPIVG, pero en el texto articulado del RD 1393/2007 se produce por una parte una clara omisión en la concreción de las materias que debían incluir una materia en todas las ramas relacionada con los derechos fundamentales y de igualdad de mujeres y hombres.

El RD 1393/2007 en el art. 3 señala que entre los principios generales que deben inspirar el diseño de los nuevos títulos, los planes de estudios deberán tener en cuenta que cualquier actividad profesional debe realizarse: a) desde el respeto a los derechos fundamentales y de igualdad entre hombres y mujeres, debiendo incluirse, en los planes de estudios en los que proceda, enseñanzas relacionadas con dichos derechos.

Se puede observar una contradicción evidente en el contenido del propio $\mathrm{RD}$ porque si señala que en todas las profesiones (cualquier actividad profesional) se deben tener en cuenta, por qué después hace referencia a que sólo en los planes de estudio en que proceda.

No obstante, la cuestión sería determinar si el RD 1393/2007 debía haber tenido en cuenta toda la legislación que se ha desarrollado en estos años en relación con la enseñanza universitaria, al efecto de desarrollar y concretar el contenido de los grados que, de acuerdo con la LOM-LOU, las directrices deben ser fijadas por el gobierno. Es cierto que la LOM-LOU no señala ninguna directriz y deja a la libertad del gobierno que fije las mismas, e incluso que pueda incrementar la participación de las respectivas universidades en el diseño de los planes de estudio, reforzando la autonomía universitaria. Pero en esta materia no sólo cabe invocar la LOM-LOU sino todas y cada una de las leyes que han regulado algunos aspectos de la enseñanza superior y concretamente la LOMPIVG y la LOI. Y como se ha señalado anteriormente, la LOMPIVG estable una obligación en la medida que señala que la docencia, la formación y la investigación deben incluir la igualdad de género, por ello el RD al desarrollar los criterios sobre los que deben ordenase las enseñazas deja sin efecto los mandatos de la LOMPIVG. Puede que el gobierno entienda que ésta es una materia que obliga a la inclusión en los planes de estudio a las 
propias universidades, pero aun en este caso, se debía haber establecido algunas garantías en el propio RD 1393/2007 para su cumplimiento. No ocurre lo mismo con la LOI dado que esta, en su art. 25, no establece obligatoriedad sino que habla de que las administraciones públicas promoverán las enseñanzas en materia de igualdad entre mujeres y hombres en los planes de estudio que procedan, y puede que el gobierno entienda que en ninguno de los nuevos planes proceda, cosa totalmente cuestionable desde el punto de vista político, pero desde luego imposible desde el punto de vista jurídico dado que existe un mandato claro sobre el tema.

A través de este análisis se puede afirmar que el RD 1393/2007 contraviene la ley, y podría darse el caso que todos y cada uno de los planes de estudio, si cumplen estrictamente con los criterios fijados por el RD 1393/2007, no tengan en cuenta ninguna materia que tenga relación con la igualdad. Sería deseable que no ocurriera tal caso, pero en el peor de los escenarios, podría ser que todos los nuevos planes de estudio estuvieran fuera de la ley o, en el mejor de los casos, sólo aquellos que no hubieran introducido ninguna de estas materias.

\section{Autonomía universitaria y estudios de género}

La autonomía universitaria es un derecho fundamental reconocido en la constitución cuyo fundamento y justificación, de acuerdo con la STC 26/1987, «... está...en el respeto a la libertad académica, es decir a la libertad de enseñanza, estudio e investigación. La protección de estas libertades frente a las injerencias externas constituye la razón de ser de la autonomía universitaria» (FJ 4).

La conceptuación como derecho fundamental no excluye las limitaciones que al mismo imponen otros derechos fundamentales o «...la existencia de un sistema universitario nacional que exige instancias coordinadoras; ni tampoco las limitaciones propias del servicio público que desempeña» (STC 26/87, FJ4).

La autonomía universitaria, es un derecho de configuración legal y la ley de universidades (LOM-LOU) es el cauce sobre el que se concreta y garantiza el derecho. Pero, como ha referido el TC, el derecho a la autonomía es ejercido con sumisión a la constitución y a las leyes.

Partiendo de estos pronunciamientos, la cuestión radica en preguntarse qué deben hacer las universidades en relación a la elaboración de los nuevos planes de estudio y los estudios de género. Como se ha señalado anteriormente, las leyes obligan a incluir y fomentar los estudios de género en el ámbito de la educación superior, y concretamente la LOMPIVG establece un mandato a 
las universidades ${ }^{40}$ para que incluyan en todos los ámbitos académicos la formación en igualdad de género y no discriminación. Por tanto, no cabe duda de que las universidades, a la hora de elaborar los planes de estudio, deben incorporar los estudios de género. Sin embargo, se podría argumentar que el gobierno no ha desarrollado, en el RD 1393/2007 de 29 de octubre por el que se establece la ordenación de las enseñanzas universitarias oficiales, las directrices necesarias para que las universidades puedan cumplir este mandato. No obstante, el RD 1393/2007 antes citado deja un gran margen a la autonomía universitaria para establecer este tipo de enseñanzas. Así, en el art.12 del RD 1393/2007 establece que los planes de estudio tendrán 240 créditos, que contendrán toda la formación teórica y práctica que el estudiante deba adquirir: aspectos básicos de la rama de conocimiento, materias obligatorias y optativas, seminarios, prácticas externas, trabajos dirigidos, trabajo fin de Grado u otras actividades formativas.

De los 240 créditos, las directrices del Gobierno expresadas en el RD sólo comprometen 36 créditos por lo que deja un margen bastante amplio para el desarrollo de la autonomía universitaria posibilitando la inclusión de otras materias diferentes a las señaladas en el RD 1393/2007. Así, el apartado 5 del art. 12 del RD 1393/2007 señala que el plan de estudios deberá contener un mínimo de 60 créditos de formación básica de los que al menos 36 estarán vinculados a algunas de las materias que figuran en el anexo II del RD 1393/2007 para cada una de las ramas de conocimiento, pero el resto de créditos hasta 60 pueden estar configurados por materias básicas de la misma u otras ramas de conocimiento de las incluidas en el anexo II o por otras materias siempre que se justifique su carácter básico para la formación inicial del estudiantado o su carácter transversal. Además quedan en el ámbito de la autonomía universitaria el diseño de los 130 créditos restantes.

Por tanto, las universidades tienen un amplio margen para cumplir con la legislación en esta materia. Cabe delimitar claramente la obligación de las universidades de cumplir las leyes con independencia de que el gobierno al dictar el RD 1393/2007 no haya desarrollado correctamente el contenido de las mismas, sobre todo de la ley contra la violencia de género. Lo que nos llevaría a afirmar que cualquiera de los planes de estudio que no incorpore asignaturas vinculadas a la materia de estudios de género o no obligue a desarrollar las materias de carácter tranversal, se aparta del contenido de las leyes y por tanto puede ser susceptible de impugnación por no cumplir las leyes.

40. En art. 4.7 de la LOMPIVG, establece: «Las Universidades incluirán y fomentarán en todos los ámbitos académicos la formación, docencia e investigación en igualdad de género y no discriminación de forma transversal». 


\section{ANEXO I CONCLUSIONES DE LAS JORNADAS}

«Los Estudios de las Mujeres y de Género en las Universidades Valencianas:

hacia el Espacio Único Europeo de Educación Superior».

Centro de Coordinación de Estudios de Género

de las Universidades Valencianas Valencia 9-10 Mayo 2005.

Los días 9 y 10 de mayo de 2005, el Centro de Coordinación de Estudios de Género de las Universidades Valencianas ${ }^{41}$ organizó las Jornadas: «Los Estudios de las Mujeres y de Género en las Universidades Valencianas: hacia el Espacio Único Europeo de Educación Superior».

Las jornadas fueron inauguradas por el Rector de la Universidad de Valencia, Francisco Tomás, y contaron con una representación de profesorado universitario, personal de organización y gestión universitaria, profesorado de enseñanzas secundaria y primaria y estudiantes de máster y doctorado.

En el acto de inauguración, el Rector Francisco Tomás recordó que las universidades de Valencia, Alicante, Miguel Hernández y Jaume I se comprometieron públicamente el 16 de Febrero de 2004 a potenciar los estudios e investigaciones de Género en el acto de presentación y entrega del I premio de investigación «Presen Sáez de Descatllar», cuyas futuras ediciones hay que consolidar. Asimismo mencionó la Ley 9/2003 de Igualdad entre Mujeres y Hombres de la Generalitat Valenciana ${ }^{42}$, donde está prevista la financiación de actividades anuales para la promoción de asignaturas y proyectos docentes que incorporen la perspectiva de género.

41. El Centro de Coordinación de Estudios de Género de las Universidades Valencianas se constituyó el 21 de febrero de 2003 con la finalidad de impulsar y reforzar las investigaciones y los estudios de las Mujeres y del Género en la Comunidad Valenciana. Está compuesto por: Institut Universitari d'Estudis de la Dona Universitat de Valéncia (IUED); Centro de Estudios sobre la Mujer, Universidad de Alicante (CEM); Seminario Interdisciplinar de Estudios de Género, Universidad Miguel Hernández (SIEG); Seminari d'Investigació Feminista y Fundación Isonomía, Universitat Jaime I.

42. Artículo 9. Promoción en la universidad de la igualdad de oportunidades. Del Capítulo II de la ley 9/2003 de igualdad entre mujeres y hombres de la Generalitat Valenciana: «A tal efecto, el Gobierno Valenciano financiará en colaboración con las universidades valencianas, actividades anuales para la promoción de asignaturas y proyectos docentes con un enfoque de género. Promoción en la Universidad de la igualdad de oportunidades. Las universidades deben promover la implantación de asignaturas y realización de proyectos docentes que incorporen la perspectiva de género». 
Las aportaciones de las ponentes y el debate entre las asistentes dieron sobrado cumplimiento de los objetivos de las jornadas. En primer lugar, se detectaron las necesidades de formación del estudiantado universitario desde la perspectiva de género para el futuro desarrollo de su actividad profesional en cualquier área del conocimiento y de actividad. En segundo lugar, se abordó la planificación e integración de la investigación interdisciplinar con la docencia de Estudios de las Mujeres y de Género en el marco del Espacio Europeo de Educación Superior.

En la reflexión en profundidad acerca de la situación actual y las perspectivas de futuro de los Estudios de las Mujeres, Feministas y de Género en la universidad española, el balance resultó ser positivo en cuanto a la investigación pero en mucha menor medida en cuanto a la docencia.

Así, en relación a la investigación, tanto la elaboración del Libro Blanco sobre los Estudios de las Mujeres ${ }^{43}$ como, en especial, el Plan sectorial del I+D sobre los Estudios de las Mujeres y del Género, han contribuido a consolidar el potencial investigador en este ámbito. No obstante queda pendiente todavía la instauración de un programa de becas asociado a dicho plan sectorial. Por otra parte, la presencia de evaluadoras expertas en Estudios de Género en todas las instancias de evaluación de proyectos se ha desarrollado únicamente de manera parcial y no sistemática ${ }^{44}$.

Por el contrario la situación de la docencia es un tema pendiente. Si bien se introdujeron en la última reforma de los planes de estudios algunas asignaturas de carácter optativo, ello no ha ocurrido en todas las titulaciones y tampoco de forma generalizada y asumida por todas las universidades, sino por el esfuerzo individual del profesorado en determinadas áreas. Es necesario proceder a un desarrollo coordinado de asignaturas con perspectiva de género, tanto en los estudios de grado como de postgrado.

A escala europea, la adecuación del sistema educativo para profundizar en la democracia y promover la ciudadanía activa en Europa implica una re-

43. Veáse: Ballarín Domingo, P., Gallego Méndez, M.T. y Martínez Benlloch, I.: Los estudios de las Mujeres en las Universidades españolas 1975-1991. Libro Blanco, Madrid, Colección Estudios 44, Ministerio de Asuntos Sociales, Instituto de la Mujer, 1995. Véase también su posterior actualización.

44. En este aspecto España está todavía muy alejada de cumplir con el objetivo de participación de las mujeres en un $40 \%$ en todos los paneles de evaluadores, comités de expertos y grupos de investigación (Women and science: Excellence and Innovation - Gender Equality in Science, Commission of the European Communities, SEC (2005) 370, Bruselas 11.3.2005). 
estructuración completa para acomodarse a las nuevas necesidades y valores, entre los que destaca el desarrollo del principio de igualdad ${ }^{45}$.

Uno de los elementos clave en la agenda de modernización de las universidades europeas es precisamente aumentar su atractivo respondiendo adecuadamente a las necesidades que demanda la integración de los futuros graduados y graduadas en la vida profesional ${ }^{46}$.

Uno de los ejemplos más emblemáticos de la producción de conocimiento científico para la solución de problemas sociales es el problema de la violencia contra las mujeres. Así se recoge en la Ley Orgánica 1/2004 de medidas de protección integral contra la violencia de género. Por una parte, la igualdad entre hombres y mujeres queda declarada como uno de los fines del sistema educativo y como un principio de calidad del mismo. Por otro lado, de acuerdo con el articulo 4, «Las universidades incluirán y fomentarán en todos los ámbitos académicos la formación, docencia e investigación en igualdad de género y no discriminación de forma transversal».

Asimismo, la transversalidad implica a todos los niveles de enseñanza, desde las enseñanzas obligatorias hasta la enseñanza universitaria de grado y postgrado. Así como la formación profesional y la formación de personas adultas. La transmisión de contenidos sin sesgo de género en la formación continua, tanto de profesorado universitario como no universitario es asimismo esencial para el éxito de un cambio en profundidad de las actitudes sexistas que provocan la discriminación y la violencia contra las mujeres.

A este fin se decidió establecer contactos institucionales con las/os asistentes a las jornadas con la finalidad de constituir una red permanente de aprendizaje e intercambio de experiencias, así como la posibilidad de colaboración en futuros proyectos de investigación y en acciones de intervención inmediata a todos los niveles de enseñanza para la transformación de los roles de género.

Finalmente, la adaptación de las enseñanzas de grado y de postgrado es una ocasión óptima para implementar las recomendaciones de la Comisión Europea, en particular las que hacen referencia al principio de igualdad y dotar de contenido a las diferentes leyes que a escala nacional y autonómica están ya en vigor.

45. Progress towards the Lisbon objectives in education and training, Commission of the European Communities, SEC (2005) 419, Bruselas 22.3.2005.

46. Mobilising the brainpower of Europe: enabling universities to make their full contribution to the Lisbon Strategy, Communication from the Commssionn of the European Communities, SEC (2005) 518, Bruselas, COM (2005) 152 final. 
Así, se decidió actuar a dos niveles de estudios (el grado y el postgrado) y en dos instancias (la nacional y la autonómica).

Primero. En cuanto a los estudios de grado, de acuerdo con el artículo 4.7 de la ley Orgánica 1/2004 de medidas de protección integral contra la violencia de género creemos necesario la puesta en marcha de una asignatura troncal en todas las titulaciones. Para ello se instó a AUDEM (Asociación Universitaria de Estudios de la Mujer) a organizar un encuentro con la finalidad de realizar una propuesta concreta para cada titulación. Dicha propuesta se elevaría al Ministerio de Educación para que la incluyera en las directrices generales de cada titulación.

Segundo. En cuanto a los estudios de postgrado, independientemente de trabajar por postgrados amplios, flexibles y coordinados entre las diferentes Comunidades Autónomas, se decidió trasladar a la Dirección General de la Mujer y al Observatorio de Género de la Generalitat Valenciana, la propuesta de financiación y puesta en marcha con carácter inmediato de un postgrado de «Género y Políticas de Igualdad», como proyecto piloto.

Tercero.- Para poder impartir la docencia se requiere que el profesorado tenga formación en estudios de feminismo y del género, por ello las universidades deben establecer los mecanismos necesarios (comisiones de personas expertas) para el cumplimiento de este requisito, a la vez que procurar la formación del profesorado que tenga interés en impartir docencia, en esta materia y carezca de la formación necesaria.

Cuarto.- Entendemos necesario que la investigación universitaria esté presidida por un análisis feminista y no androcéntrico desde una perspectiva transversal y para ello es necesario incorporar personas expertas en esta materia, de cada uno de los ámbitos científicos y en todos aquellos organismos públicos dedicados a la concesión y evaluación de proyectos y actividad investigadora.

\begin{abstract}
ANEXO II
Encuentro andaluz sobre Feminismo y Universidad La proyección política de los Estudios de Género

Baeza, 30 de junio - 1 de julio de 2005
\end{abstract}

\title{
Conclusiones
}

Mesa de Trabajo: «Presente y Futuro de los Estudios de Género: Nuevos paradigmas cognitivos, nuevas realidades de intervención» 
- Se reconoce la dispersión actual de los Estudios de Género. Ante ello, son necesarias redes de difusión de los estudios, actividades, etc. que en temas de género se realizan, al menos en las Universidades andaluzas y entre las investigadoras que desarrollan su labor en Andalucía.

- Se insiste en la importancia de la formación feminista de las nuevas generaciones universitarias. Para ello, se ve la necesidad de continuar e incrementar la formación y la especialización en los estudios de género e igualdad mediante la oferta de másteres, cursos, estudios de tercer ciclo, títulos de expert@s, etc. de esa especialidad.

- Se considera necesario el tratamiento transversal de los estudios de género, introduciendo la perspectiva de género y feminista en aquellas asignaturas de los planes de estudios en las que esto sea posible. Tal medida no excluye que se sigan ofertando y potenciando asignaturas, materias, títulos, etc. específicos de temas de género.

- Se considera que en la actualidad existe coyuntura favorable para conseguir que los nuevos planes de estudios incorporen materias de género, dado que se está en pleno proceso de elaboración de nuevos planes comunes al ámbito europeo. Se entiende que ese cambio ha de venir de las instancias superiores y que, en consecuencia, es necesaria una voluntad política de jerarquías. Pero tal evidencia no debe hacernos olvidar que también es imprescindible una presión desde las bases académicas.

- Se considera la necesidad de que la formación en género y feminismo no quede reducida al ámbito universitario, sino que ha de extenderse a otros niveles de la enseñanza. Para ello, y como estrategia complementaria, se ve la necesidad de realizar una revisión de los textos utilizados en los niveles educativos de primaria y secundaria, así como un control severo de los medios de comunicación, especialmente de la televisión pública, que en ocasiones oferta programas infantiles de alto contenido machista. En este último sentido se apunta la posibilidad de crear un Comité de sabi@s que orienten a la clase política al respecto.

Baeza, 1 de julio de 2005 


\begin{abstract}
ANEXO III
La Incorporación de los Estudios Feministas, de Mujeres y de Género a la Enseñanza Universitaria
\end{abstract}

Durante el año 2005 se ha realizado una serie de reuniones acerca de la incorporación de los estudios feministas, de mujeres y de género a la enseñanza universitaria a las cuales han asistido numerosas personas provenientes de diferentes universidades así como de otros ámbitos sociales y profesionales. La reunión celebrada en Oñati en septiembre partió de las conclusiones elaboradas en los encuentros anteriores celebrados en Valencia en mayo y en Baeza en julio. A partir de las reflexiones planteadas en estos encuentros acordamos hacer públicas las siguientes consideraciones y propuestas.

En la actualidad estamos viviendo una coyuntura especialmente importante en lo que respecta a la incorporación de los estudios feministas, de mujeres y de género a la enseñanza universitaria. Por un lado, el proceso de construcción del Espacio Europeo de Enseñanza Superior impulsado a partir de la Declaración de Bolonia marca la necesidad de reforma y elaboración de nuevos planes de estudio de todas las titulaciones universitarias. Por otro lado, existe un marco legal dirigido a que las universidades desarrollen de forma transversal formación, docencia e investigación en torno a la igualdad de género y la no discriminación desde la aprobación de la Ley Orgánica 1/2004 de Medidas de Protección Integral contra la Violencia de Género así como de otras Leyes de Igualdad aprobadas en diversas Comunidades Autónomas. Es importante tener en cuenta asimismo, que actualmente se está elaborando el borrador de la Ley de Igualdad que previsiblemente aprobará el Parlamento español en los próximos meses.

En este contexto, consideramos que este es un momento clave para que las universidades asuman dos cuestiones fundamentales:

1. La incorporación institucional de modelos concretos de estudios feministas, de mujeres y de género a la enseñanza universitaria.

2. Un compromiso firme y explícito a favor de la igualdad y la no discriminación entre los sexos que se materialice en la creación de estructuras y asignación de recursos orientados a tal fin dentro de la propia universidad.

Por lo que respecta a la incorporación de los estudios, existen diversos modelos posibles que lejos de ser excluyentes, responden a necesidades complementarias y se basan en la diversidad y pluralidad de las universidades: 
1. Creación de un Grado como titulación específica de Estudios de Género orientado fundamentalmente a la formación de la figura profesional de Agente de Igualdad.

2. Creación de Posgrados específicos orientados a la especialización en materia de estudios de género en distintas áreas de conocimiento.

3. Oferta de asignaturas específicas (troncales y optativas) adaptadas a cada una de las titulaciones universitarias dirigidas a introducir la perspectiva de los estudios de género en las distintas disciplinas.

4. Adaptación de los programas de diversas asignaturas introduciendo temas en aquellos en los que se considere pertinente.

Por todo ello, pedimos a las instituciones públicas con competencia en materia de enseñanza superior y en particular a las universidades que incorporen modelos concretos de estudios feministas, de mujeres y de género a la enseñanza universitaria y que hagan explícito su compromiso en favor de la igualdad y la no discriminación entre los sexos dentro y fuera de la propia universidad.

\section{ANEXO IV}

Las profesoras universitarias especialistas en Estudios de Género, reunidas en el ler. Congreso «Los estudios sobre las mujeres, de género y feministas. Grados y postgrados en el Espacio Europeo de Educación Superior» los días 20 y 21 de noviembre de 2006 :

Reconocemos y damos la bienvenida al esfuerzo del que se hace eco la LOU por establecer un mayor equilibrio entre mujeres y hombres en los distintos ámbitos de gestión y representación universitaria, pero entendemos que esta apuesta no es suficiente para potenciar los cambios en las relaciones de género que nuestra sociedad requiere y que deben acompañarse de las oportunas directrices dirigidas a la transformación de los conocimientos que se construyen y difunden.

Concretamente, el Artículo 35 de la LOU dice «El Gobierno establecerá las directrices y las condiciones para la obtención de los títulos universitarios de carácter oficial y con validez en todo el territorio nacional».

Pues bien, de acuerdo con las consideraciones anteriores:

Solicitamos que en las directrices que el Gobierno elabore y remita a las universidades para el diseño de todas las titulaciones de grado se incorporen materias especializadas en Estudios de Género. 
Entendemos que estas materias se deben articular como asignaturas troncales que incorporen a todas las disciplinas y titulaciones las aportaciones de los Estudios de Género.

Consideramos que ante la inexistencia de área de conocimiento de Estudios de Genero y con objeto de garantizar la calidad de los conocimientos que se impartan, el Ministerio debe arbitrar las medidas para que una comisión experta en esta temática evalúe la idoneidad del perfil de las/os docentes que hayan de impartir las citadas asignaturas.

Igualmente consideramos conveniente que entre los indicadores de evaluación de la calidad de las Universidades públicas y privadas, se incluyan aquellos parámetros que den cuenta de la aplicación efectiva del principio de igualdad entre mujeres y hombres.

Madrid, 21 de noviembre de 2006 